\title{
On the accuracy of approximate techniques for the evaluation of lightning electromagnetic fields along a mixed propagation path
}

\author{
Abdolhamid Shoory, ${ }^{1}$ Abdenabi Mimouni, ${ }^{2}$ Farhad Rachidi, ${ }^{1}$ Vernon Cooray, ${ }^{3}$ \\ and Marcos Rubinstein ${ }^{4}$
}

Received 6 July 2010; revised 30 November 2010; accepted 21 December 2010; published 3 March 2011.

[1] In this paper we review simplified analytical expressions derived by Wait using the concept of attenuation function for the analysis of the propagation of lightning-radiated electromagnetic fields over a mixed propagation path (vertically stratified ground).

Two different formulations proposed by Wait that depend on the relative values of ground surface impedances are discussed. It is shown that both formulations give nearly the same results for the time domain electric field. However, depending on the values of the normalized surface impedance for each ground section, the use of one of the two formulations is computationally more efficient. The accuracy of the Wait formulations was examined taking as reference full-wave simulations obtained using the finite difference time domain technique. It is shown that Wait's simplified formulas are able to reproduce the distant field peak and waveshape with a good accuracy.

Citation: Shoory, A., A. Mimouni, F. Rachidi, V. Cooray, and M. Rubinstein (2011), On the accuracy of approximate techniques for the evaluation of lightning electromagnetic fields along a mixed propagation path, Radio Sci., 46, RS2001, doi:10.1029/2010RS004480.

\section{Introduction}

[2] The study of wave propagation along a vertically stratified ground goes back to the early works of Millington [1949], Kirke [1949], Suda [1954], and Bremmer [1954]. In particular, using an integral equation formulation, Bremmer [1954] showed that the semiempirical work of Millington [1949] was valid for a wide range of frequencies and ground parameters. The recovery effect, namely, the increase of the wave steepness when it passes from a poor conducting ground to a high conducting ground, can also be satisfactorily reproduced by Millington's approach.

\footnotetext{
${ }^{1}$ Electromagnetic Compatibility Laboratory, Swiss Federal Institute of Technology, Lausanne, Switzerland.

${ }^{2}$ Laboratoire de Génie Physique, Ibn Khaldoun University, Tiaret, Algeria.

${ }^{3}$ Ångström Laboratory, Division for Electricity and Lightning Research, Uppsala University, Uppsala, Sweden.

${ }^{4}$ Department of Electrical Engineering and Computer Science, University of Applied Sciences of Western Switzerland, Yverdonles-Bains, Switzerland.

Copyright 2011 by the American Geophysical Union. 0048-6604/11/2010RS004480
}

Wait [1956] extended the formulation of Bremmer [1954] to low and medium frequencies by using the compensation theorem [e.g., Monteath, 1973] and the mutual impedances between two vertical dipoles located above ground. A set of simulation results and related curves were presented and discussed by Wait and Householder [1957] and Wait and Walters [1963a]. They showed that the simulation results are in acceptable agreement with measured data. Further confirmation of the technique was then given by King et al. [1973], who reported excellent agreement between the Wait formulation [Wait, 1956] and experimental data obtained from reduced scale experiments carried out at the frequency of 4.765 GHz.

[3] The main idea behind the Wait's formulation is the use of the surface impedance concept which is defined as the ratio of electric and magnetic fields tangential to the ground surface [Hill and Wait 1980] in the same way as for either a homogeneous or a stratified ground [see Wait, 1998].

[4] The application of these studies to the analysis of lightning electromagnetic field propagation was first carried out by Cooray and Ming [1994] and Cooray and Perez [1994]. It was specifically shown by Cooray and Ming [1994] that when the propagation is over a mixed 
(sea-land) path whose land portion is less than a few hundred meters long, the attenuation of the vertical electric field is not significant. However, the peak time derivative of the vertical electric field would suffer significant attenuation of about $40 \%$ in propagating more than a few tens of meters over the land portion. Such a theoretical development was also used by Cooray and Ming [1994] for the interpretation of the experimental data by Willett et al. [1988, 1990] and Cooray [1986] for a measuring station that was located a few tens of meters from the sealand interface. Good agreement was found between measured waveforms and theoretical predictions for vertical electric fields and their time derivatives. The errors in predicting peak current and peak current derivative from peak vertical electric field and peak vertical electric field derivative were also discussed by Cooray and Ming [1994]. Such a theoretical analysis was later used by Michishita et al. [1996] to estimate the ground electrical parameters from measured horizontal and vertical electric fields radiated by distant lightning.

[5] For the case of a homogenous ground, the accuracy of approximate formulae has been recently studied by Cooray [2008, 2005] through comparisons with exact solutions of the Sommerfeld integrals. Delfino et al. [2007, 2008], using an efficient algorithm for the evaluation of the exact expressions for both the aboveground and underground fields generated by a lightning discharge, tested the validity of the Cooray-Rubinstein formula [Rubinstein, 1996; Cooray, 1992] for the computation of the horizontal electric field of lightning above a homogeneous ground. More recently, Shoory et al. [2010] studied the accuracy of simplified formulations for the case of a horizontally stratified ground, using as a reference results obtained using the finite difference time domain (FDTD) technique.

[6] To the best of the authors' knowledge, the accuracy of the simplified analytical expressions for the case of a mixed path (vertically stratified) ground has been only studied using a limited number of reduced scale experiments and in the frequency domain [King et al., 1973], and no systematic analysis is available in the literature.

[7] The aim of this paper is to examine the accuracy of the simplified formulations taking as reference full-wave simulations obtained using the FDTD technique.

[8] The paper is organized as follows. In section 2, two simplified formulations based on the concept of attenuation function are reviewed and discussed. Time domain simulations for the far field are presented and compared to the reference case of a homogeneous ground. In section 3, the accuracy of the simplified formulations is tested versus full-wave simulations obtained using the FDTD technique. Conclusions are given in section 4 .

\section{Simplified Approaches for the Lightning Far Field Above a Vertically Stratified Ground}

\subsection{Formulations}

[9] Denoting the vertical electric field at ground level over a perfectly conducting ground at a distance $\rho$ from the channel base in the time domain as $e_{z p}$ and in the frequency domain as $E_{z p}$, the expression for the vertical electric field in the frequency domain, over a vertically stratified ground as shown in Figure 1 can be written as [see Cooray, 2003, chapter 7]

$$
E_{z, m i x}=E_{z p} F_{m i x}
$$

where $F_{\text {mix }}$ is the attenuation function for a vertically stratified ground (mixed path). The expression for the corresponding time domain vertical electric field can be represented using the convolution integral as

$$
e_{z, \text { mix }}=\int_{0}^{t} e_{z p}(\tau) f_{\text {mix }}(t-\tau) d \tau
$$

where $f_{\text {mix }}$ is the inverse Fourier transform of the attenuation function $F_{m i x}$. Note that the attenuation function corresponds to a dipole located at the lower end of the channel as proposed by Cooray and Ming [1994].

[10] Two different expressions for the mixed-path ground attenuation function are given by Wait and Householder [1957], Wait [1961, 1974], and Wait and Walters [1963a, $1963 \mathrm{~b}]$

$F_{\text {mix }}(\rho)=F_{1}(\rho)-\sqrt{\frac{\gamma_{0} \rho}{2 \pi}}\left[\Delta_{2}-\Delta_{1}\right] \int_{0}^{d l} \frac{F_{1}(\rho-x) F_{2}(x)}{\sqrt{x(\rho-x)}} d x$

$$
F_{\text {mix }}(\rho)=F_{2}(\rho)-\sqrt{\frac{\gamma_{0} \rho}{2 \pi}}\left[\Delta_{1}-\Delta_{2}\right] \int_{0}^{\rho-d l} \frac{F_{2}(\rho-x) F_{1}(x)}{\sqrt{x(\rho-x)}} d x
$$

where $F_{1}(x)$ and $F_{2}(x)$ are the attenuation functions of each section of the ground represented as a function of the horizontal distance from the source to the observation point, defined as follows:

$$
F_{n}(x)=1-j \sqrt{\pi p_{n}} e^{-p_{n}} \operatorname{erfc}\left(j \sqrt{p_{n}}\right)
$$




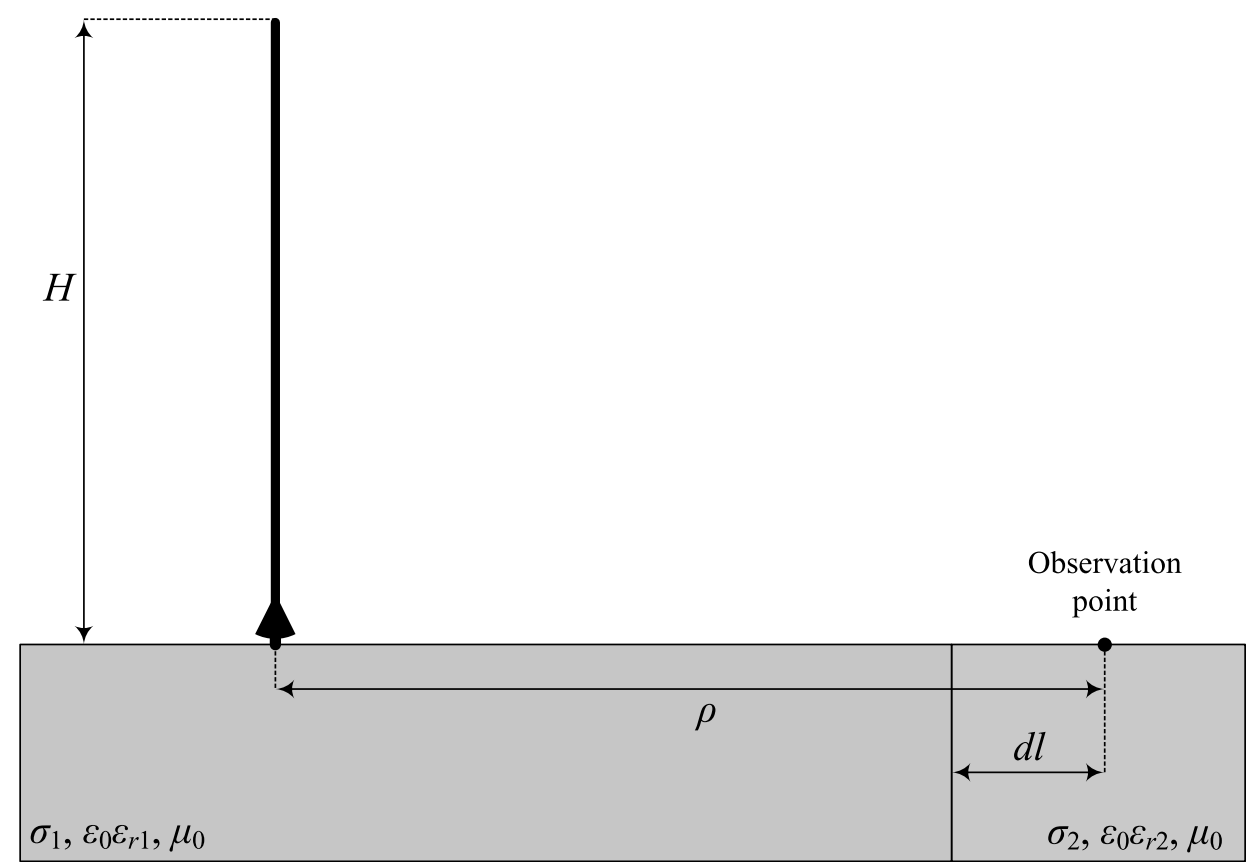

Figure 1. Propagation of lightning-radiated electromagnetic field over a two-layer vertically stratified ground.

where

$$
p_{n}(x)=-0.5 \gamma_{0} x \Delta_{n}^{2}
$$

in which $\Delta_{n}(n=1,2)$ is the normalized surface impedance of each ground section defined as [e.g., Hill and Wait, 1980]

$$
\Delta_{n}=\frac{\sqrt{j \omega \varepsilon_{0}\left[\sigma_{n}+j \omega \varepsilon_{0}\left(\varepsilon_{r n}-1\right)\right]}}{\sigma_{n}+j \omega \varepsilon_{0} \varepsilon_{r n}}
$$

Hill and Wait [1981] have suggested to use (3) when $\left|\Delta_{2}\right|<\left|\Delta_{1}\right|$, and to use (4) when $\left|\Delta_{2}\right|>\left|\Delta_{1}\right|$. They have also shown that there are singularities in the integrands of (3) and (4), and they have provided the necessary steps to remove them. The results are given below

$$
\begin{aligned}
F_{\text {mix }}(\rho)= & F_{1}(\rho)-\sqrt{\frac{\gamma_{0} \rho}{2 \pi}}\left[\Delta_{2}-\Delta_{1}\right] \\
& \cdot\left\{2 \sqrt{\frac{\delta}{\rho}} F_{1}(\rho)+\int_{\delta}^{d l} \frac{F_{1}(\rho-x) F_{2}(x)}{\sqrt{x(\rho-x)}} d x\right\} \\
F_{\text {mix }}(\rho)= & F_{2}(\rho)-\sqrt{\frac{\gamma_{0} \rho}{2 \pi}}\left[\Delta_{1}-\Delta_{2}\right] \\
& \cdot\left\{2 \sqrt{\frac{\delta}{\rho}} F_{2}(\rho)+\int_{\delta}^{\rho-d l} \frac{F_{2}(\rho-x) F_{1}(x)}{\sqrt{x(\rho-x)}} d x\right\}
\end{aligned}
$$

where $\delta$ is a small distance over which one can assume the attenuation functions to be constant.

[11] Note that throughout this paper, whenever we encounter square roots of complex quantities, we will choose the roots on the upper Riemann sheet [see Chew, 1995]. It is also to be emphasized that the simplified formulations above are obtained assuming the stationary phase approximation or, equivalently, the line-of-sight assumption for wave propagation between the channel base and the observation point. In this regard, the formulations are valid for either straight or circular boundary line between the two media in Figure 1.

\subsection{Numerical Results}

[12] For the time domain simulations, we adopted the MTLE model [Nucci et al., 1988; Rachidi and Nucci, 1990] with a current decay constant $\lambda=2 \mathrm{~km}$ and a return stroke speed of $v=1.5 \times 10^{8} \mathrm{~m} / \mathrm{s}$. The channel base current is that of Rachidi et al. [2001], which corresponds to a typical subsequent return stroke. The parameters of each ground layer according to Figure 1 are shown in Table 1. Time domain simulation results of the vertical electric field at ground level and at a distance of $\rho=$ $100 \mathrm{~km}$ from the channel base for a mixed path ground with $d l=25 \mathrm{~km}$ and with the electrical parameters shown in Table 1 are shown in Figures $2 \mathrm{a}$ and $2 \mathrm{~b}$. The curves are obtained using the two previously introduced formulations (8) and (9). Figure 2 also includes two curves 
Table 1. Considered Parameters for the Mixed Path Ground ${ }^{\mathrm{a}}$

\begin{tabular}{lcc}
\hline & Case 1 & Case 2 \\
\hline$\sigma_{1}(\mathrm{~S} / \mathrm{m})$ & 0.001 & 4 \\
$\varepsilon_{\mathrm{r} 1}(\mathrm{~S} / \mathrm{m})$ & 10 & 30 \\
$\sigma_{2}$ & 4 & 0.001 \\
$\varepsilon_{\mathrm{r} 2}$ & 30 & 10 \\
\hline
\end{tabular}

${ }^{\mathrm{a}}$ See Figure 1.

of the limiting cases obtained when the ground is homogeneous with the parameters of medium 1 and medium 2, respectively. It is seen that the two formulations predict almost the same results for the vertical electric field. It can also be observed that the vertical electric field over a vertically stratified ground falls in between the two curves of limiting homogenous ground cases confirming the conclusions given by Cooray [2003, chapter 7]. Further, by virtue of the reciprocity theorem, the two formulations are transparent to the direction of wave propagation from one medium to the other. In other words, by interchanging the lightning channel and the observation point, the wave at the observation point does not change. As mentioned in section 2.1 , it is computationally more efficient in this case for the curves of Figure 2 a to use (8) instead of (9) and for the curves of Figure $2 b$ to use (9) instead of (8).

\section{Test and Validation of the Simplified Formula}

[13] In this section, the reviewed simplified formulations for a vertically stratified ground are validated against the FDTD simulation technique. The details of the technique are given by Yee [1966]. The procedure is essentially the same as that used by Mimouni et al. [2007a, 2007b, 2008] and Shoory et al. [2010]. The simulation domain of the FDTD technique is shown in Figure 3. The technique solves the two Maxwell's curl equations by means of the so-called Yee discretization scheme [Yee, 1966]. In this regard, one needs to solve only for the vertical and horizontal electric fields and the azimuthal magnetic field due to the axial symmetry of the problem (the axial symmetry implies a circular boundary between the two media in Figure 1). The spatial and temporal derivatives are represented simply using the first-order finite differences. More details on the technique are given, for instance, by Mimouni et al. [2007a, 2007b, 2008].

[14] Two sets of simulations have been performed for a distance of $\rho=10 \mathrm{~km}$ according to the two different mixed path arrangements of Table 1 . In the FDTD simulations, a value for the time step of 2 ns was considered. The overall time interval was set to $T_{\max }=50 \mu \mathrm{s}$. This corresponds to 25,000 time steps.

[15] The spatial discretization interval was chosen to be $2 \mathrm{~m}$. According to Figure 3, the simulation domain was truncated using the first order Mur absorbing boundary conditions at $\rho_{\max }=11 \mathrm{~km}, z_{\max }=7.5 \mathrm{~km}$, and $z_{\min }=100 \mathrm{~m}$. Making use of the axial symmtery of the problem (leftmost boundary coinciding with the $\mathrm{z}$ axis), this corresponds to $5500 \times 3800$ spatial cells. The simulations were carried out on a 64 bit computational platform having 32 GB of available memory.

[16] Note that a shorter distance of $\rho=10 \mathrm{~km}$ (instead of $\rho=100 \mathrm{~km}$ in Figure 2) was used in this section due the lack of computational resources necessary for simulations in the FDTD technique. In fact, with the available resources, the smallest spatial discretization interval for $\rho=100 \mathrm{~km}$ satisfying the Courant stability criterion [Courant et al., 1967] is $20 \mathrm{~m}$, which imposes significant numerical dispersion on the predicted benchmark waveforms. In fact, for the case of propagation along a lossy half-space, numerical dispersion might occur because of the dependence of wave propagation speed on frequency.
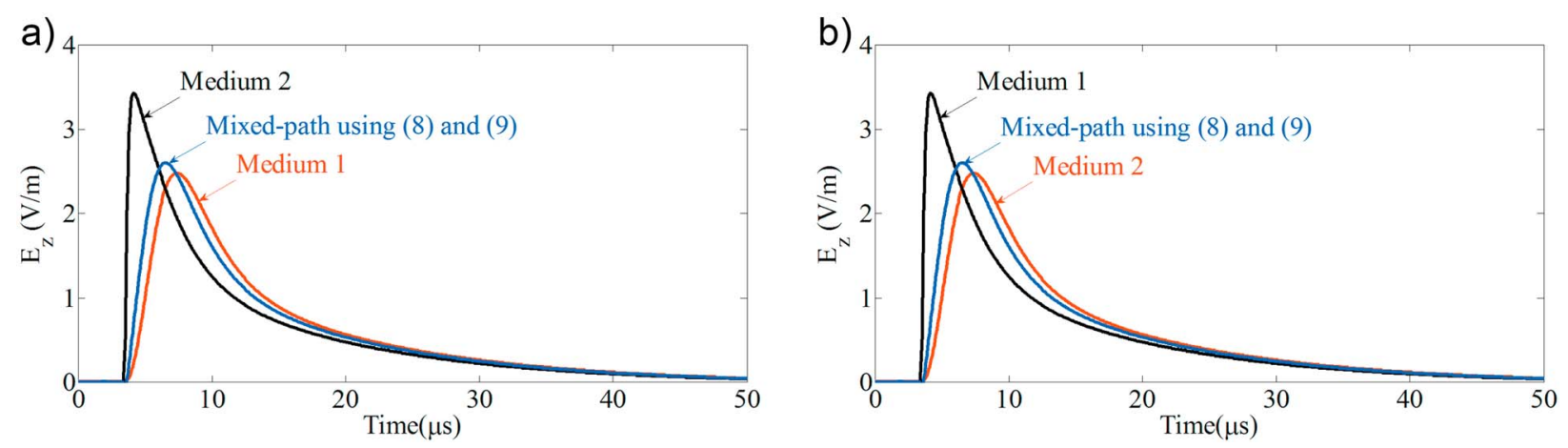

Figure 2. Vertical electric field at ground level at a distance of $\rho=100 \mathrm{~km}$ from the channel base over mixed path ground with $d l=25 \mathrm{~km}$ obtained using the two formulations of (8) and (9) for (a) case 1 and (b) case 2 according to Table 1 . 


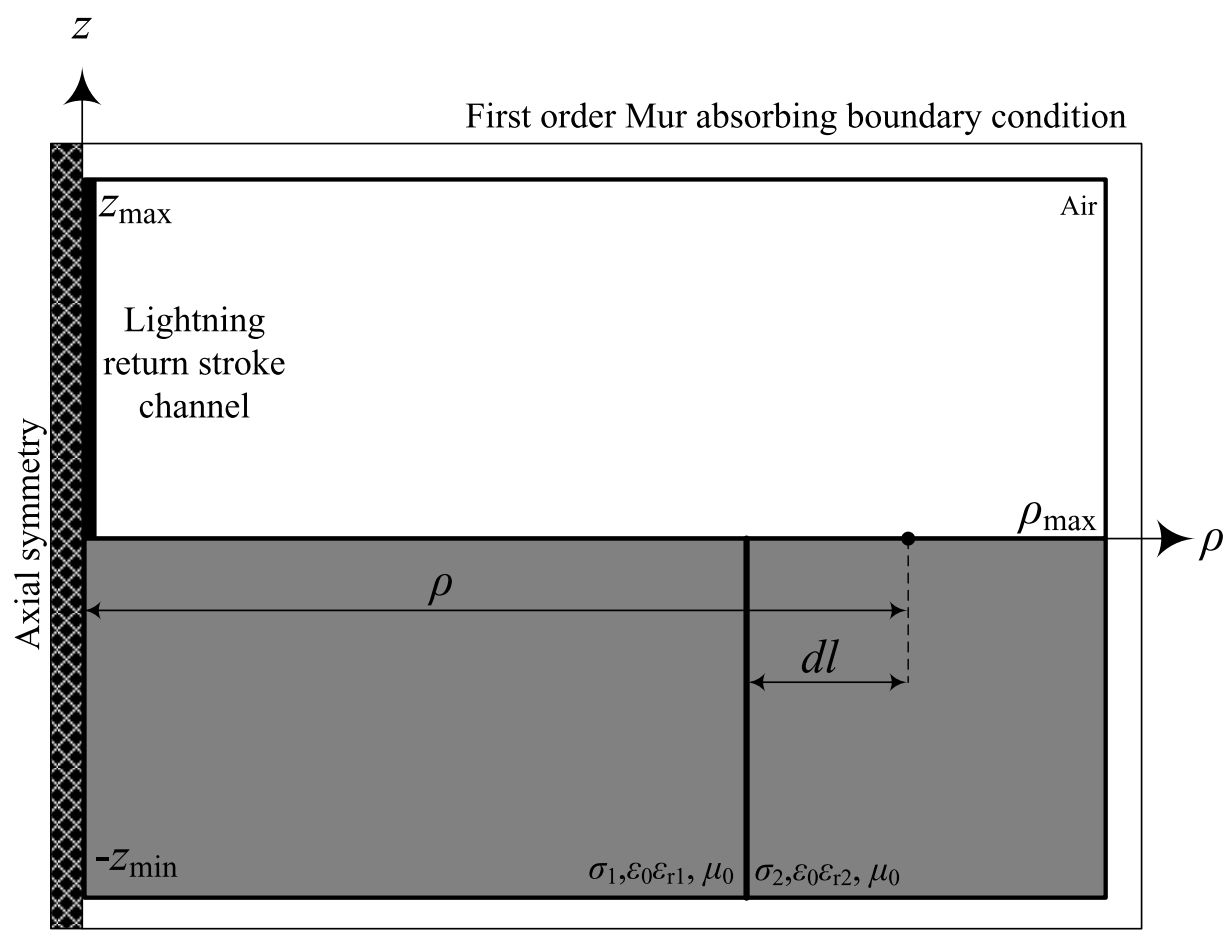

Figure 3. Side view of the simulation domain of the FDTD technique used for the validation of the simplified approaches. We used $\rho_{\max }=11 \mathrm{~km}, z_{\max }=7.5 \mathrm{~km}$, and $z_{\min }=100 \mathrm{~m}$.
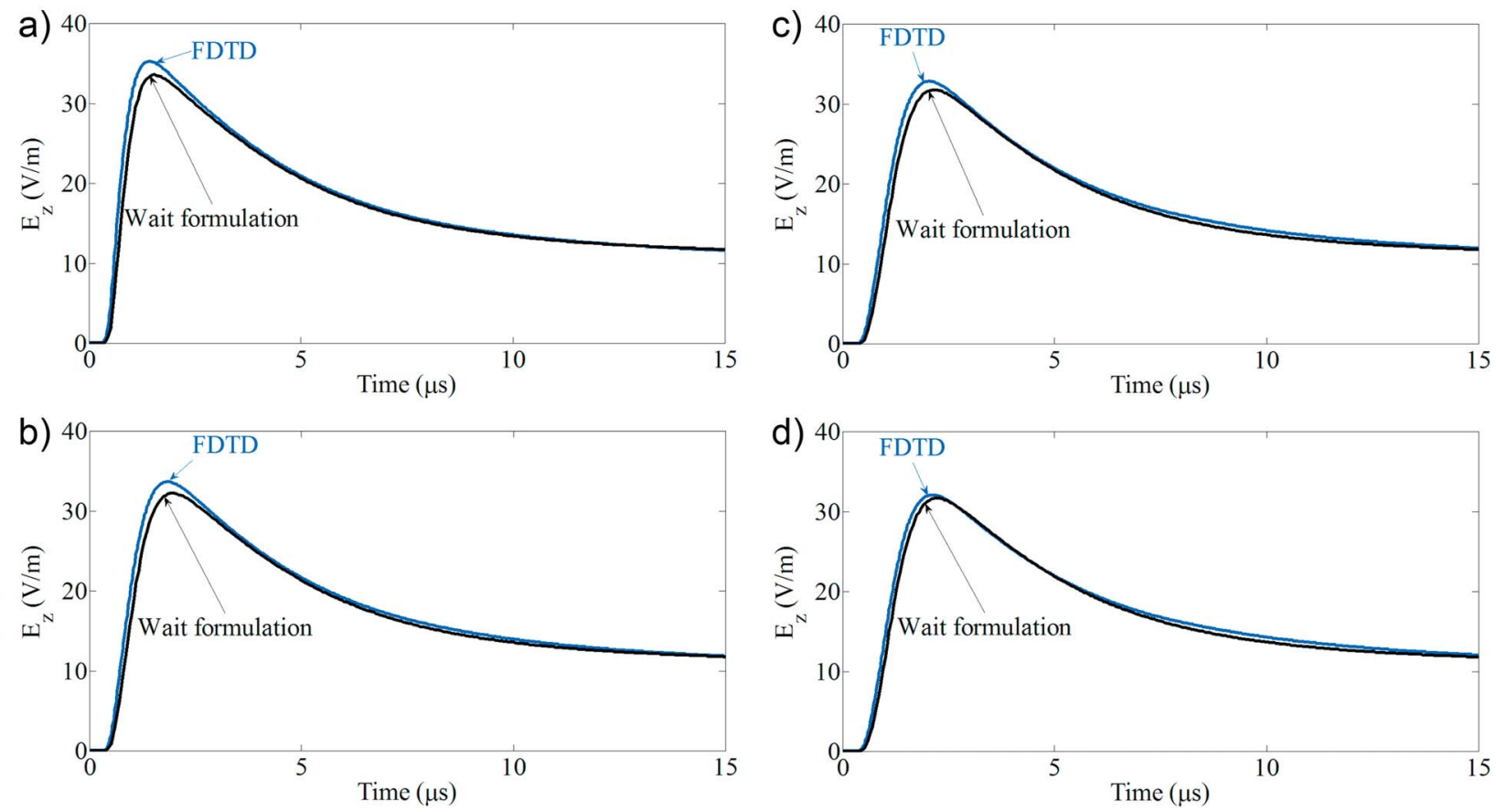

Figure 4. Vertical electric field at the surface of a mixed-path ground at a distance of $\rho=10 \mathrm{~km}$ for case 1 in Table 1 for (a) $d l=7.5 \mathrm{~km}$, (b) $d l=2.5 \mathrm{~km}$, (c) $d l=0.5 \mathrm{~km}$, and (d) $d l=0.1 \mathrm{~km}$. 

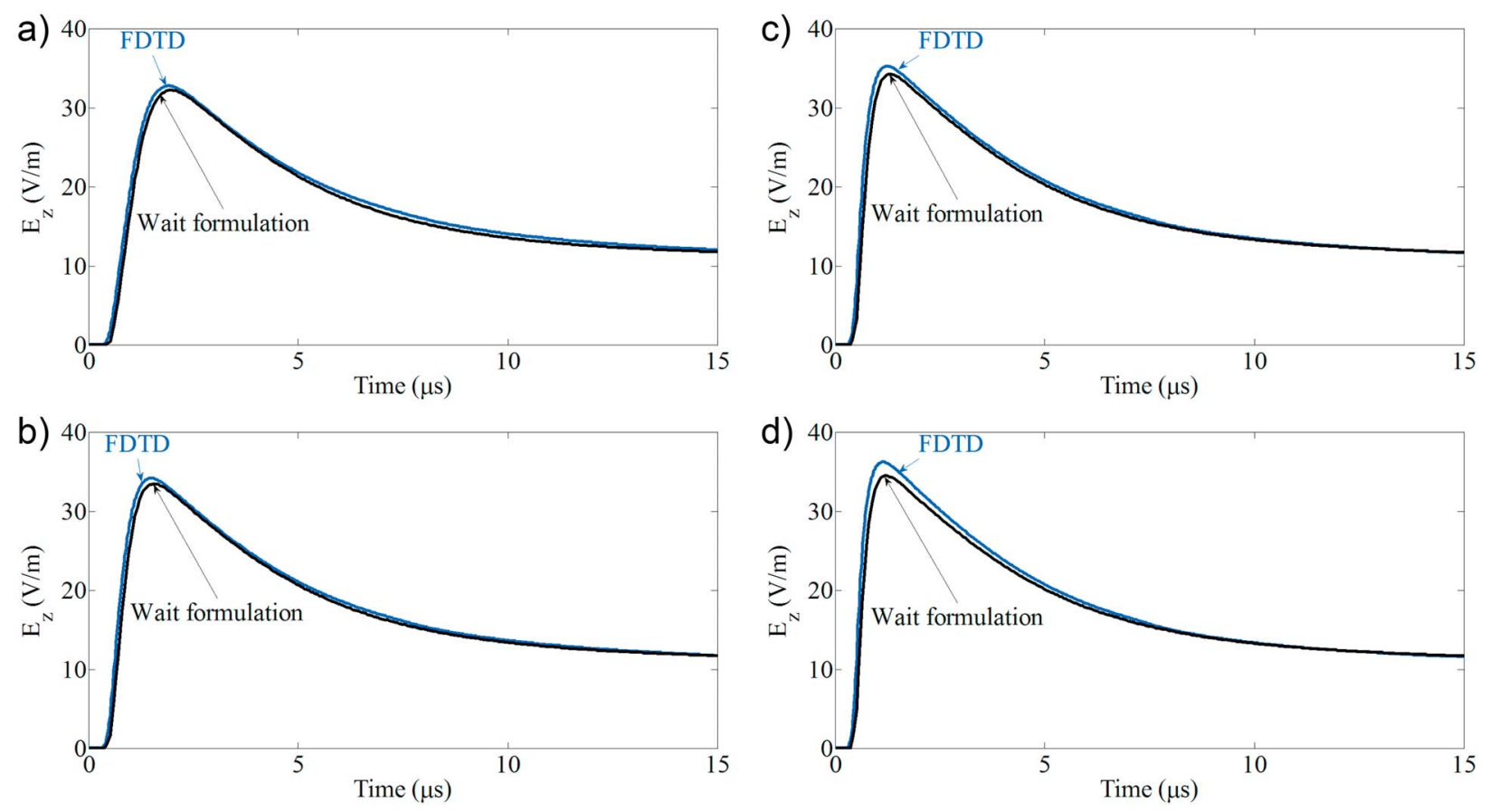

Figure 5. Vertical electric field at the surface of a mixed-path ground at a distance of $\rho=10 \mathrm{~km}$ for case 2 in Table 1 for (a) $d l=7.5 \mathrm{~km}$, (b) $d l=2.5 \mathrm{~km}$, (c) $d l=0.5 \mathrm{~km}$, and (d) $d l=0.1 \mathrm{~km}$.

In general, the bigger the spatial discretization interval, the larger the numerical dispersion. While for an observation point at $\rho=10 \mathrm{~km}$ the results obtained using a spatial step of $2 \mathrm{~m}$ are free from numerical dispersion, this is not the case for an observation point at $\rho=100 \mathrm{~km}$ with spatial step of $20 \mathrm{~m}$. In order to minimize this effect, a finer mesh should be considered.

[17] Simulation results of a vertical electric field at a distance of $\rho=10 \mathrm{~km}$ from the channel base at ground level according to case 1 in Table 1 are shown in Figure 4 for four different values of $d l$, namely, 7.5, 2.5, 0.5, and $0.1 \mathrm{~km}$. The same results for case 2 are shown in Figure 5. It can be seen that Wait's formulation appears to be quite accurate in reproducing the vertical electric field waveforms.

[18] Table 2 presents the values of the field peaks and zero-to-peak risetimes predicted by Wait's formulation and by the FDTD method. It can be seen that Wait's formulation can predict field peaks with an error of less than $4.8 \%$ and field risetimes with an error of less than $18.0 \%$.

\section{Conclusions}

[19] Simplified analytical expressions derived by Wait using the concept of attenuation function for the analysis of the propagation of lightning-radiated electromagnetic fields over a vertically stratified ground were reviewed. Two different formulations proposed by Wait that depend on the relative values of the ground surface impedances were discussed. Both formulations give nearly the same results for the time domain electric field. However, depending on the values of the normalized surface impedance for each ground section, the use of one of the two formulations is computationally more efficient.

Table 2. Peak and Risetimes of the Vertical Electric Field Predicted Using Wait's Formulation and FDTD Simulation

\begin{tabular}{|c|c|c|c|c|c|c|}
\hline \multirow[b]{2}{*}{$\begin{array}{c}d l \\
(\mathrm{~km})\end{array}$} & \multicolumn{3}{|c|}{ Peak $(\mathrm{V} / \mathrm{m})$} & \multicolumn{3}{|c|}{ Risetime $(\mu \mathrm{s})$} \\
\hline & Wait & FDTD & $\begin{array}{c}\text { Error } \\
(\%)\end{array}$ & Wait & FDTD & $\begin{array}{c}\text { Error } \\
(\%)\end{array}$ \\
\hline & & & Case 1 & & & \\
\hline 7.5 & 33.5 & 35.2 & 4.8 & 1.3 & 1.1 & 18 \\
\hline 2.5 & 32.2 & 33.6 & 4.2 & 1.6 & 1.5 & 6.3 \\
\hline 0.5 & 31.8 & 32.8 & 3.0 & 1.8 & 1.7 & 5.9 \\
\hline 0.1 & 31.6 & 32.1 & 1.6 & 2 & 1.8 & 10 \\
\hline & & & Case 2 & & & \\
\hline 7.5 & 32.2 & 32.7 & 1.5 & 1.6 & 1.6 & 0.0 \\
\hline 2.5 & 33.6 & 34.1 & 1.5 & 1.3 & 1.2 & 8.3 \\
\hline 0.5 & 34.4 & 35.3 & 2.5 & 1.0 & 0.9 & 11.1 \\
\hline 0.1 & 34.7 & 36.2 & 4.1 & 0.9 & 0.8 & 12.5 \\
\hline
\end{tabular}


[20] The accuracy of the Wait formulations was examined taking as reference full-wave simulations obtained using the finite difference time domain (FDTD) technique. It was shown that Wait's simplified formulas are able to reproduce the distant field peak and waveshape with a good accuracy. At a distance of $10 \mathrm{~km}$, the maximum observed error for the peak electric field was $4.8 \%$. The maximum error for its zero-to-peak risetime was $18 \%$. It is worth noting that the simplified formulations were originally developed for the radiation component of the field and for the case where the interface is far enough from both the lightning channel and the observation point for the radiation component to be dominant. More work is needed to evaluate the accuracy of Wait's formulations for observation points located at closer distances to the lightning channel and/or to the interface.

\section{References}

Bremmer, H. (1954), The extension of the Sommerfeld's formula for the propagation of radio waves over a flat earth to different conductivities of the soil, Physica, 20(1-6), 441-460, doi:10.1016/S0031-8914(54)80061-9.

Chew, W. C. (1995), Waves and Fields in Inhomogeneous Media, IEEE Press, New York.

Cooray, V. (1986), A novel method to identify the radiation fields produced by positive return strokes and their submicrosecond structure, J. Geophys. Res., 91(D7), 7907-7911, doi:10.1029/JD091iD07p07907.

Cooray, V. (1992), Horizontal fields generated by return strokes, Radio Sci., 27(4), 529-537, doi:10.1029/91RS02918.

Cooray, V. (2003), The Lightning Flash, Inst. of Electr. Eng., London.

Cooray, V. (2005), On the validity of several approximate theories used in quantifying the propagation effects on lightning generated electromagnetic fields, paper presented at VIII Simpósio Internacional de Proteção Contra Descargas, Inst. of Electrotech. and Energy, Univ. of Sao Paulo, Sao Paulo, Brazil, 21-25 Nov.

Cooray, V. (2008), On the accuracy of several approximate theories used in quantifying the propagation effects on lightning generated electromagnetic fields, IEEE Trans. Antennas Propag., 56(7), 1960-1967, doi:10.1109/TAP.2008. 924680.

Cooray, V., and Y. Ming (1994), Propagation effects on the lightning-generated electromagnetic fields for homogeneous and mixed sea-land paths, J. Geophys. Res., 99(D5), 10,641-10,652, doi:10.1029/93JD03277.

Cooray, V., and H. Perez (1994), Propagation effects on the first return stroke radiation fields: Homogenous paths and mixed two section paths, paper presented at 22nd International Conference Lightning Protection, Hung. Electrotech. Assoc., Budapest, 19-23 Sept.
Courant, R., K. Friedrichs, and H. Lewy (1967), On the partial difference equations of mathematical physics, IBM J. Res. Develop., 11, 215-234, doi:10.1147/rd.112.0215.

Delfino, F., R. Procopio, F. Rachidi, and C. A. Nucci (2007), An algorithm for the exact evaluation of the underground lightning electromagnetic fields, IEEE Trans. Electromagn. Compat., 49(2), 401-411, doi:10.1109/TEMC.2007.897127.

Delfino, F., R. Procopio, and M. Rossi (2008), Lightning return stroke current radiation in presence of a conducting ground: 1. Theory and numerical evaluation of the electromagnetic fields, J. Geophys. Res., 113, D05110, doi:10.1029/ 2007JD008553.

Hill, D. A., and J. R. Wait (1980), Ground wave attenuation function for a spherical earth with arbitrary surface impedance, Radio Sci., 15(3), 637-643, doi:10.1029/RS015i003p00637.

Hill, D. A., and J. R. Wait (1981), HF ground wave propagation over mixed land, sea, and sea-ice paths, IEEE Trans. Geosci. Remote Sens., GE-19(4), 210-216.

King, R. J., S. H. Cho, and D. H. Jaggard (1973), Height-gain experimental data for ground wave propagation: 2. Heterogeneous paths, Radio Sci., 8, 17-22, doi:10.1029/ RS008i001p00017.

Kirke, H. L. (1949), Calculation of ground wave field strength over a composite land path, Proc. IRE, 37, 489-496, doi:10.1109/JRPROC.1949.232644.

Michishita, K., M. Ishii, and J.-I. Hojo (1996), Measurement of horizontal electric fields associated with distant cloudto-ground strokes, J. Geophys. Res., 101(D2), 3861-3867, doi:10.1029/95JD03069.

Millington, G. (1949), Ground wave propagation over an inhomogeneous smooth Earth, part 1, Proc. IEE, 96, 53-64, doi:10.1049/pi-3.1949.0013.

Mimouni, A., F. Rachidi, and Z. Azzouz (2007a), Electromagnetic environment in the immediate vicinity of a lightning return stroke, J. Lightning Res., 2, 64-75.

Mimouni, A., F. Delfino, R. Procopio, and F. Rachidi (2007b), On the computation of underground electromagnetic fields generated by lightning: A comparison between different approaches, paper presented at IEEE PES PowerTech, Electrosuisse, Lausanne, Switzerland, 1-5 July.

Mimouni, A., F. Rachidi, and Z. Azzouz (2008), A finitedifference time-domain approach for the evaluation of electromagnetic fields radiated by lightning to tall structures, J. Electrost., 66, 504-513, doi:10.1016/j.elstat.2008.05.002.

Monteath, G. D. (1973), Application of the Electromagnetic Reciprocity Principle, Pergamon, Oxford, U. K.

Nucci, C. A., C. Mazzetti, F. Rachidi, and M. Ianoz (1988), On lightning return stroke models for LEMP calculations, paper presented at 19th International Conference on Lightning Protection, Austrian Electrotec. Assoc., Graz, Austria, May.

Rachidi, F., and C. A. Nucci (1990), On the Master, Uman, Lin, Standler and the modified transmission line lightning return stroke current models, J. Geophys. Res., 95(D12), 20,389-20,393, doi:10.1029/JD095iD12p20389. 
Rachidi, F., W. Janischewskyj, A. M. Hussein, C. A. Nucci, S. Guerrieri, B. Kordi, and J. S. Chang (2001), Current and electromagnetic field associated with lightning return strokes to tall towers, IEEE Trans. Electromagn. Compat., 43(3), 356-367, doi:10.1109/15.942607.

Rubinstein, M. (1996), An approximate fomula for the caluclation of the horizontal electric field from lightning at close, intermediate, and long range, IEEE Trans. Electromagn. Compat., 38, 531-535, doi:10.1109/15.536087.

Shoory, A., A. Mimouni, F. Rachidi, V. Cooray, R. Moini, and S. H. H. Sadeghi (2010), Validity of simplified approaches for the evaluation of lightning electromagnetic fields above a horizontally stratified ground, IEEE Trans. Electromagn. Compat., 52(3), 657-663, doi:10.1109/TEMC.2010.2045229.

Suda, K. (1954), Field strength calculations-new method for mixed paths, Wireless Eng., 31, 249.

Wait, J. R. (1956), Mixed path ground wave propagation: 1. Short distances, J. Res. Natl. Inst. Stand. Technol., 57(1), $1-15$.

Wait, J. R. (1961), On the theory of mixed-path ground-wave propagation on a spherical earth, J. Res. Natl. Inst. Stand. Technol., 65D(4), 401-410.

Wait, J. R. (1974), Recent analytical investigations of electromagnetic ground wave propagation over inhomogeneous earth models, Proc. IEEE, 62(8), 1061-1072, doi:10.1109/ PROC.1974.9570.

Wait, J. R. (1998), The ancient and modern history of EM ground-wave propagation, Antennas Propag. Mag., 40(5), 7-24, doi:10.1109/74.735961.

Wait, J. R., and J. Householder (1957), Mixed-path ground wave propagation: 2. Larger distances, J. Res. Natl. Inst. Stand. Technol., 59(1), 19-26.

Wait, J. R., and L. Walters (1963a), Curves for ground wave propagation over mixed land and sea paths, IEEE Trans. Antennas Propag., 11(1), 38-45, doi:10.1109/TAP.1963.1137979.
Wait, J. R., and L. Walters (1963b), Correction to "Curves for ground wave propagation over mixed land and sea paths," IEEE Trans. Antennas Propag., 11(3), 329, doi:10.1109/ TAP.1963.1138017.

Willett, J. C., V. P. Idone, R. E. Orville, C. Leteinturier, A. Eybert Berard, L. Barret, and E. P. Krider (1988), An experimental test of the 'transmission-line model' of electromagnetic radiation from triggered lightning return strokes, J. Geophys. Res., 93(D4), 3867-3878, doi:10.1029/JD093iD04p03867.

Willett, J. C., J. C. Bailey, C. Leteinturier, and E. P. Krider (1990), Lightning electromagnetic radiation field spectra in the interval from 0.2 to $20 \mathrm{MHz}, J$. Geophys. Res., 95(D12), 20,367-20,387, doi:10.1029/JD095iD12p20367.

Yee, K. S. (1966), Numerical solution of initial boundary value problems involving Maxwell's equations in isotropic media, IEEE Trans. Antennas Propag., 14, 302-307, doi:10.1109/ TAP.1966.1138693

V. Cooray, Ångström Laboratory, Division for Electricity and Lightning Research, Uppsala University, S-75121 Uppsala, Sweden. (vernon.cooray@angstrom.uu.se)

A. Mimouni, Laboratoire de Génie Physique, Ibn Khaldoun University, Tiaret 14000, Algeria. (abdenbi.mimouni@gmail. com)

F. Rachidi and A. Shoory, Electromagnetic Compatibility Laboratory, Swiss Federal Institute of Technology, Lausanne 1015, Switzerland. (farhad.rachidi@epfl.ch; abdolhamid. shoory@epfl.ch)

M. Rubinstein, Department of Electrical Engineering and Computer Science, University of Applied Sciences of Western Switzerland, CH-1400 Yverdon-les-Bains, Switzerland. (marcos.rubinstein@heig-vd.ch) 